\title{
Article \\ Uniqueness of Solutions of the Generalized Abel Integral Equations in Banach Spaces
}

\author{
Chenkuan $\mathrm{Li}^{1, *(\mathbb{D})}$ and Hari M. Srivastava $2,3,4,5$ (D) \\ 1 Department of Mathematics and Computer Science, Brandon University, Brandon, MB R7A 6A9, Canada \\ 2 Department of Mathematics and Statistics, University of Victoria, Victoria, BC V8W 3R4, Canada; \\ harimsri@uvic.ca \\ 3 Department of Medical Research, China Medical University Hospital, China Medical University, \\ Taichung 40402, Taiwan \\ 4 Department of Mathematics and Informatics, Azerbaijan University, 71 Jeyhun Hajibeyli Street, \\ Baku AZ1007, Azerbaijan \\ 5 Section of Mathematics, International Telematic University Uninettuno, I-00186 Rome, Italy \\ * Correspondence: lic@brandonu.ca
}

check for

updates

Citation: Li, C.; Srivastava, H.M

Uniqueness of Solutions of the Generalized Abel Integral Equations in Banach Spaces. Fractal Fract. 2021, 5, 105. https://doi.org/10.3390/ fractalfract 5030105

Academic Editor: Paul Eloe

Received: 4 August 2021

Accepted: 28 August 2021

Published: 31 August 2021

Publisher's Note: MDPI stays neutral with regard to jurisdictional claims in published maps and institutional affiliations.

Copyright: (c) 2021 by the authors. Licensee MDPI, Basel, Switzerland. This article is an open access article distributed under the terms and conditions of the Creative Commons Attribution (CC BY) license (https:// creativecommons.org/licenses/by/ $4.0 /)$.
Abstract: This paper studies the uniqueness of solutions for several generalized Abel's integral equations and a related coupled system in Banach spaces. The results derived are new and based on Babenko's approach, Banach's contraction principle and the multivariate Mittag-Leffler function. We also present some examples for the illustration of our main theorems.

Keywords: Riemann-Liouville fractional integral; Banach's fixed point theorem; Babenko's approach; Wright's generalized Bessel function; multivariate Mittag-Leffler function

\section{Introduction}

Let $T>0$. The space $L[0, T]$ is given by

$$
L[0, T]=\left\{u(x):\|u\|=\int_{0}^{T}|u(x)| d x<\infty\right\} .
$$

Clearly, $L[0, T]$ is a Banach space. The product space $L[0, T] \times L[0, T]$ (which is also a Banach space) is defined as follows:

$$
L[0, T] \times L[0, T]=\{(u(x), v(x)): u(x), v(x) \in L[0, T]\},
$$

with the norm given by

$$
\|(u, v)\|=\|u\|+\|v\| .
$$

The Riemann-Liouville fractional integral $I^{\alpha}$ of order $\alpha \in \mathbb{R}^{+}$is defined for the function $u(x)$ by (see [1,2]):

$$
\left(I^{\alpha} u\right)(x)=\frac{1}{\Gamma(\alpha)} \int_{0}^{x}(x-t)^{\alpha-1} u(t) d t
$$

In particular, we have

$$
\left(I^{0} u\right)(x)=u(x)
$$

Let $0 \leqq \alpha_{0}<\alpha_{i}$ for $i=1,2, \cdots, m$ and $\alpha_{0} \leqq \alpha$. In this paper, we begin to construct an explicit solution in $L[0, T]$ to the following Abel's integral equation by using Babenko's approach and the multivariate Mittag-Leffler function:

$$
I^{\alpha_{0}} u(x)+\sum_{i=1}^{m} a_{i} I^{\alpha_{i}} u(x)=I^{\alpha} f(x),
$$


where each $a_{i}(i=1,2, \cdots, m)$ is a constant and $f(x) \in L[0, T]$. We then further investigate the uniqueness of solutions in $L[0, T]$ for the following nonlinear Abel's integral equation by using Banach's fixed point theorem:

$$
I^{\alpha_{0}} u(x)+\sum_{i=1}^{m} a_{i} I^{\alpha_{i}} u(x)=I^{\alpha} g(x, u(x)),
$$

where $g$ is a mapping from $[0, T] \times \mathbb{R}$ to $\mathbb{R}$ and satisfies certain conditions. Finally, the sufficient conditions are given for the uniqueness of solutions in the product space $L[0, T] \times$ $L[0, T]$ to the associated system given by

$$
\left\{\begin{array}{l}
I^{\alpha_{0}} u(x)+\sum_{i=1}^{m} a_{i} I^{\alpha_{i}} u(x)=I^{\alpha} g_{1}(x, u(x), v(x)) \\
I^{\beta_{0}} v(x)+\sum_{i=1}^{m} b_{i} I^{\beta_{i}} v(x)=I^{\beta} g_{2}(x, u(x), v(x)),
\end{array}\right.
$$

where $g_{1}$ and $g_{2}$ are mappings from $[0, T] \times \mathbb{R}^{2}$ to $\mathbb{R}, 0 \leqq \beta_{0} \leqq \beta$ and $\beta_{0}<\beta_{i}$ for all $i=1,2, \cdots, m$. Equations (1)-(3) are new and, to the best of our knowledge, have never been investigated earlier.

The single-term (for $m=1$ ) Equation (1) turns out to be

$$
u(x)+a_{1} I^{\alpha_{1}} u(x)=f(x) \quad\left(\alpha=\alpha_{0}=0\right),
$$

which is the classical Abel's integral equation of the second kind with the following solution given by Hille and Tamarkin (see, for details [3]; see also [4-6]):

$$
u(x)=f(x)-a_{1} \int_{0}^{x}(x-t)^{\alpha_{1}-1} E_{\alpha_{1}, \alpha_{1}}\left(-a_{1}(x-t)^{\alpha_{1}}\right) f(t) d t,
$$

where $E_{\alpha, \beta}(z)$ given by

$$
E_{\alpha, \beta}(z)=\sum_{j=0}^{\infty} \frac{z^{j}}{\Gamma(\alpha j+\beta)} \quad(\alpha, \beta>0),
$$

is the two-parameter Mittag-Leffler function.

The above solution can also be easily deduced by the Laplace transform. Indeed,

$$
\mathcal{L}\left(u(x)+a_{1} I^{\alpha_{1}} u(x)\right)=\mathcal{L} f(x)=\tilde{f}(s)
$$

infers that

$$
\tilde{u}(s)+\frac{a_{1}}{s^{\alpha_{1}}} \tilde{u}(s)=\tilde{f}(s) .
$$

Hence, we have

$$
\tilde{u}(s)=\left(1-\frac{a_{1}}{a_{1}+s^{\alpha_{1}}}\right) \tilde{f}(s) .
$$

Using the formula (1.80) from [7]

$$
\int_{0}^{\infty} e^{-s t} t^{\alpha_{1}-1} E_{\alpha_{1}, \alpha_{1}}\left(-a_{1} t^{\alpha_{1}}\right) d t=\frac{1}{a_{1}+s^{\alpha_{1}}},
$$

we arrive at

$$
\begin{aligned}
u(x) & =f(x)-a_{1} x^{\alpha_{1}-1} E_{\alpha_{1}, \alpha_{1}}\left(-a_{1} x^{\alpha_{1}}\right) * f(x) \\
& =f(x)-a_{1} \int_{0}^{x}(x-t)^{\alpha_{1}-1} E_{\alpha_{1}, \alpha_{1}}\left(-a_{1}(x-t)^{\alpha_{1}}\right) f(t) d t
\end{aligned}
$$


where $\phi * \psi$ denotes the Laplace convolution given by

$$
(\phi * \psi)(x)=\int_{0}^{x} \phi(x-t) \psi(t) d t .
$$

On the other hand, Babenko's approach is a potentially powerful tool for solving differential, integral and integro-differential equations by treating integral operators like variables. The method itself is similar to the Laplace transform method while dealing with such equations with constant coefficients, but it can be used in other cases as well, such as handling integral equations with variable coefficients (see $[8,9])$. To demonstrate this method, we are going to solve Equation (4) with Babenko's approach. Clearly, Equation (4) becomes

$$
\left(1+a_{1} I^{\alpha_{1}}\right) u(x)=f(x) .
$$

Therefore, we get

$$
\begin{aligned}
u(x) & =\left(1+a_{1} I^{\alpha_{1}}\right)^{-1} f(x)=\sum_{k=0}^{\infty}(-1)^{k}\left(a_{1} I^{\alpha_{1}}\right)^{k} f(x) \\
& =f(x)+\sum_{k=0}^{\infty}(-1)^{k+1}\left(a_{1} I^{\alpha_{1}}\right)^{k+1} f(x) \\
& =f(x)-a_{1} \sum_{k=0}^{\infty}\left(-a_{1}\right)^{k} \frac{1}{\Gamma\left(\alpha_{1} k+\alpha_{1}\right)} \int_{0}^{x}(x-t)^{\alpha_{1} k+\alpha_{1}-1} f(t) d t \\
& =f(x)-a_{1} \int_{0}^{x}(x-t)^{\alpha_{1}-1} \sum_{k=0}^{\infty}\left(-a_{1}\right)^{k} \frac{1}{\Gamma\left(\alpha_{1} k+\alpha_{1}\right)}(x-t)^{\alpha_{1} k} f(t) d t \\
& =f(x)-a_{1} \int_{0}^{x}(x-t)^{\alpha_{1}-1} E_{\alpha_{1}, \alpha_{1}}\left(-a_{1}(x-t)^{\alpha_{1}}\right) f(t) d t .
\end{aligned}
$$

We now recall Wright's generalized Bessel Function $\varphi(\beta, \delta ; z)$ defined as follows:

$$
\varphi(\beta, \delta ; z)=\sum_{j=0}^{\infty} \frac{z^{j}}{j ! \Gamma(\beta j+\delta)} \quad(\beta, \delta>0) .
$$

We also define

$$
S_{m}^{\mu}\left(x ; z_{1}, \cdots, z_{m} ; \beta_{1}, \cdots, \beta_{m}\right)=\left(h_{1} * h_{2} * \cdots * h_{m}\right)(x),
$$

where

$$
\begin{gathered}
h_{k}=h_{k}(x)=x^{\mu_{k}-1} \phi\left(\beta_{k}, \mu_{k} ; z_{k} x^{\beta_{k}}\right) \quad\left(x, \beta_{k}>0 ; z_{k} \in \mathbb{R}\right), \\
\mu=\sum_{k=1}^{m} \mu_{k}, \quad \mu_{k}>0 .
\end{gathered}
$$

Let

$$
\begin{aligned}
& G_{m}^{\mu}\left(x ; \gamma_{1}, \cdots, \gamma_{m} ; \beta_{1}, \cdots, \beta_{m}\right) \\
& \quad=\int_{0}^{\infty} e^{-t} S_{m}^{\mu}\left(x ; \gamma_{1} t, \cdots, y_{m} t ; \beta_{1}, \cdots, \beta_{m}\right) d t
\end{aligned}
$$

and

$$
w_{\mu}(x)=G_{m}^{\mu-\alpha_{0}}\left(x ;-a_{1}, \cdots,-a_{m} ; \alpha_{1}-\alpha_{0}, \cdots, \alpha_{m}-\alpha_{0}\right) \quad\left(\mu>\alpha_{0}\right) .
$$


In 2013, Pskhu [10] constructed an explicit solution for the following Abel's integral equation (which is a special case of the Equation (1)):

$$
I^{\alpha_{0}} u(x)+\sum_{i=1}^{m} a_{i} I^{\alpha_{i}} u(x)=I^{\alpha_{0}} f(x) \quad(f \in L[0, T]),
$$

as follows:

$$
u(x)=D_{0, x}^{\mu}\left(f * w_{\mu}\right)(x) \quad\left(\mu>\alpha_{0}\right),
$$

where the solution $u(x)$ is independent of the parameter $\mu$ and

$$
D_{0, x}^{\mu} \phi(x):= \begin{cases}\frac{1}{\Gamma(-\mu)} \int_{0}^{x} \phi(t)(x-t)^{-\mu-1} d t=I^{-\mu} \phi(x) & (\mu<0) \\ \phi(x) & (\mu=0) \\ \frac{d^{n}}{d x^{n}}\left\{I^{n-\mu} \phi(x)\right\} & (n-1<\mu \leqq n ; n \in \mathbb{N}),\end{cases}
$$

$\mathbb{N}$ being the set of positive integers.

We would also like to add that Gorenflo and Luchko [11] established an explicit solution to the following generalized Abel integral equation of the second kind, which was based on a modification of the Mikusiński operational calculus and the Mittag-Leffler function of several variables (see, for details, [12]):

$$
u(x)-\sum_{j=1}^{m} a_{j} I^{\alpha_{j} \mu} u(x)=f(x) \quad\left(\alpha_{j}>0 ; m \geqq 1 ; \mu>0 ; x>0\right),
$$

which is also a special case of Equation (1).

There are many analytic and numerical studies on Abel's integral equation and its variants in distribution, as well as the existence and uniqueness of the corresponding solutions by using fixed point theorems [7,8,13-15]. For example, Brunner et al. [16] considered numerical solutions of Abel's integral equation of the second kind:

$$
u(x)=f(x)+\int_{0}^{x}(x-t)^{-\alpha} \kappa(x, t, u(t)) d t \quad(x \in[0, T]),
$$

where $0<\alpha<1$ and $f \in C[0, T]$, and the kernel $\kappa$ is continuous on $\mathbb{S} \times \mathbb{R}$, with

$$
\mathbb{S}=\{(t, s): 0 \leqq s \leqq t \leqq T\},
$$

and satisfies the Lipschitz conditions in the third argument.

The multivariate Mittag-Leffler function was studied by (among others) Hadid and Luchko [17] for solving linear fractional differential equations with constant coefficients by applying the operational calculus:

$$
\begin{aligned}
& E_{\left(\alpha_{1}, \cdots, \alpha_{m}\right), \beta}\left(z_{1}, \cdots, z_{m}\right) \\
& \quad=\sum_{k=0}^{\infty} \sum_{k_{1}+\cdots+k_{m}=k}\left(\begin{array}{c}
k \\
k_{1}, \cdots, k_{m}
\end{array}\right) \frac{z_{1}^{k_{1}} \cdots z_{m}^{k_{m}}}{\Gamma\left(\alpha_{1} k_{1}+\cdots+\alpha_{m} k_{m}+\beta\right)},
\end{aligned}
$$

where $\alpha_{i}>0(i=1,2, \cdots, m)$ and $\beta>0$.

\section{A Set of Main Results}

In this section, we begin to establish an explicit solution to Equation (1) by using Babenko's approach in [18]. 
Theorem 1. Assume that $f \in L[0, T], 0 \leqq \alpha_{0}<\alpha_{i}$ for $i=1,2, \cdots, m$ and $\alpha_{0} \leqq \alpha$. Then, Equation (1) has a unique solution in $L[0, T]$ given by

$$
\begin{gathered}
u(x)=\sum_{k=0}^{\infty}(-1)^{k} \sum_{k_{1}+\cdots+k_{m}=k}\left(\begin{array}{c}
k \\
k_{1}, \cdots, k_{m}
\end{array}\right) a_{1}^{k_{1}} \cdots a_{m}^{k_{m}} \\
\cdot I^{k_{1}\left(\alpha_{1}-\alpha_{0}\right)+\cdots+k_{m}\left(\alpha_{m}-\alpha_{0}\right)+\alpha-\alpha_{0}} f(x),
\end{gathered}
$$

where each $a_{i}(i=1,2, \cdots, m)$ is a constant.

Proof. Equation (1) becomes

$$
u(x)+\sum_{i=1}^{m} a_{i} I^{\alpha_{i}-\alpha_{0}} u(x)=I^{\alpha-\alpha_{0}} f(x),
$$

by applying the operator $D_{0, x}^{\alpha_{0}}$ to both sides of Equation (1). This implies that, by Babenko's method, we have

$$
\begin{aligned}
u(x)= & \left(1+\sum_{i=1}^{m} a_{i} I^{\alpha_{i}-\alpha_{0}}\right)^{-1} I^{\alpha-\alpha_{0}} f(x) \\
= & \sum_{k=0}^{\infty}(-1)^{k}\left(\sum_{i=1}^{m} a_{i} I^{\alpha_{i}-\alpha_{0}}\right)^{k} I^{\alpha-\alpha_{0}} f(x) \\
= & \sum_{k=0}^{\infty}(-1)^{k} \sum_{k_{1}+\cdots+k_{m}=k}\left(\begin{array}{c}
k \\
k_{1}, \cdots, k_{m}
\end{array}\right)\left(a_{1} I^{\alpha_{1}-\alpha_{0}}\right)^{k_{1}} \cdots\left(a_{m} I^{\alpha_{m}-\alpha_{0}}\right)^{k_{m}} I^{\alpha-\alpha_{0}} f(x) \\
= & \sum_{k=0}^{\infty}(-1)^{k} \sum_{k_{1}+\cdots+k_{m}=k}\left(\begin{array}{c}
k \\
k_{1}, \cdots, k_{m}
\end{array}\right) a_{1}^{k_{1}} \cdots a_{m}^{k_{m}} \\
& \quad \cdot I^{k_{1}\left(\alpha_{1}-\alpha_{0}\right)+\cdots+k_{m}\left(\alpha_{m}-\alpha_{0}\right)+\alpha-\alpha_{0}} f(x) .
\end{aligned}
$$

Let

$$
\Phi_{\gamma}(x)=\frac{x_{+}^{\gamma}}{\Gamma(\gamma)}
$$

where $\gamma \in \mathbb{R}$ and

$$
x_{+}^{\alpha}= \begin{cases}x^{\alpha} & (x>0) \\ 0 & \text { (otherwise). }\end{cases}
$$

We then find from [19] that

$$
\Phi_{\gamma_{1}} * \Phi_{\gamma_{2}}=\Phi_{\gamma_{1}+\gamma_{2}}
$$

Clearly, we have

$$
I^{k_{1}\left(\alpha_{1}-\alpha_{0}\right)+\cdots+k_{m}\left(\alpha_{m}-\alpha_{0}\right)+\alpha-\alpha_{0}} f(x)=\Phi_{k_{1}\left(\alpha_{1}-\alpha_{0}\right)+\cdots+k_{m}\left(\alpha_{m}-\alpha_{0}\right)+\alpha-\alpha_{0}} * f .
$$

Moreover, it follows from [20] that

$$
\begin{aligned}
& \left\|\Phi_{k_{1}\left(\alpha_{1}-\alpha_{0}\right)+\cdots+k_{m}\left(\alpha_{m}-\alpha_{0}\right)+\alpha-\alpha_{0}} * f\right\| \\
& \quad \leqq\left\|\Phi_{k_{1}\left(\alpha_{1}-\alpha_{0}\right)+\cdots+k_{m}\left(\alpha_{m}-\alpha_{0}\right)+\alpha-\alpha_{0}}\right\|\|f\| \\
& \quad \leqq \frac{T^{k_{1}\left(\alpha_{1}-\alpha_{0}\right)+\cdots+k_{m}\left(\alpha_{m}-\alpha_{0}\right)+\alpha-\alpha_{0}}}{\Gamma\left(k_{1}\left(\alpha_{1}-\alpha_{0}\right)+\cdots+k_{m}\left(\alpha_{m}-\alpha_{0}\right)+\alpha-\alpha_{0}+1\right)}\|f\| .
\end{aligned}
$$


This implies that

$$
\begin{aligned}
\|u(x)\| & \leqq \sum_{k=0}^{\infty} \sum_{k_{1}+\cdots+k_{m}=k}\left(\begin{array}{c}
k \\
k_{1}, \cdots, k_{m}
\end{array}\right)\left|a_{1}\right|^{k_{1}} \cdots\left|a_{m}\right|^{k_{m}} \\
& \cdot \frac{T^{k_{1}\left(\alpha_{1}-\alpha_{0}\right)+\cdots+k_{m}\left(\alpha_{m}-\alpha_{0}\right)+\alpha-\alpha_{0}}}{\Gamma\left(k_{1}\left(\alpha_{1}-\alpha_{0}\right)+\cdots+k_{m}\left(\alpha_{m}-\alpha_{0}\right)+\alpha-\alpha_{0}+1\right)}\|f\| \\
& =T^{\alpha-\alpha_{0}} E_{\left(\alpha_{1}-\alpha_{0}, \cdots, \alpha_{m}-\alpha_{0}\right), \alpha-\alpha_{0}+1}\left(\left|a_{1}\right| T^{\alpha_{1}-\alpha_{0}}, \cdots,\left|a_{m}\right| T^{\alpha_{m}-\alpha_{0}}\right)\|f\| \\
& <\infty .
\end{aligned}
$$

Hence, we get $u(x) \in L[0, T]$, and that the series on the right-hand side of Equation (5) is absolutely convergent in $L[0, T]$.

The uniqueness of the solution follows immediately from the fact that the following fractional integral equation:

$$
I^{\alpha_{0}} u(x)+\sum_{i=1}^{m} a_{i} I^{\alpha_{i}} u(x)=0,
$$

has only the zero solution by Babenko's method.

It remains to show that the series on the right-hand side of Equation (5) is a solution of Equation (1). Indeed, we have

$$
\begin{aligned}
& I^{\alpha_{0}} u(x)+ \sum_{i=1}^{m} a_{i} I^{\alpha_{i}} u(x) \\
&=\sum_{k=0}^{\infty}(-1)^{k} \sum_{k_{1}+\cdots+k_{m}=k}\left(\begin{array}{c}
k \\
k_{1}, \cdots, k_{m}
\end{array}\right) a_{1}^{k_{1}} \cdots a_{m}^{k_{m}} \cdot I^{k_{1}\left(\alpha_{1}-\alpha_{0}\right)+\cdots+k_{m}\left(\alpha_{m}-\alpha_{0}\right)+\alpha} f(x) \\
& \quad+\sum_{k=0}^{\infty}(-1)^{k} \sum_{k_{1}+\cdots+k_{m}=k}\left(\begin{array}{c}
k \\
k_{1}, \cdots, k_{m}
\end{array}\right) \sum_{i=1}^{m} a_{1}^{k_{1}} \cdots a_{i}^{k_{i}+1} \cdots a_{m}^{k_{m}} \\
& \cdot I^{k_{1}\left(\alpha_{1}-\alpha_{0}\right)+\cdots+\left(k_{i}+1\right)\left(\alpha_{i}-\alpha_{0}\right)+\cdots+k_{m}\left(\alpha_{m}-\alpha_{0}\right)+\alpha} f(x) \\
&=I^{\alpha} f(x)+\sum_{k=1}^{\infty}(-1)^{k} \sum_{k_{1}+\cdots+k_{m}=k}\left(\begin{array}{c}
k \\
k_{1}, \cdots, k_{m}
\end{array}\right) a_{1}^{k_{1}} \cdots a_{m}^{k_{m}} \\
& \cdot I^{k_{1}\left(\alpha_{1}-\alpha_{0}\right)+\cdots+k_{m}\left(\alpha_{m}-\alpha_{0}\right)+\alpha} f(x) \\
& \quad+\sum_{k=0}^{\infty}(-1)^{k} \sum_{k_{1}+\cdots+k_{m}=k}\left(\begin{array}{c}
k \\
k_{1}, \cdots, k_{m}
\end{array}\right) \sum_{i=1}^{m} a_{1}^{k_{1}} \cdots a_{i}^{k_{i}+1} \cdots a_{m}^{k_{m}} \\
& \cdot I^{k_{1}\left(\alpha_{1}-\alpha_{0}\right)+\cdots+\left(k_{i}+1\right)\left(\alpha_{i}-\alpha_{0}\right)+\cdots+k_{m}\left(\alpha_{m}-\alpha_{0}\right)+\alpha} f(x)=I^{\alpha} f(x),
\end{aligned}
$$

by noting that

$$
\begin{gathered}
\sum_{k=1}^{\infty}(-1)^{k} \sum_{k_{1}+\cdots+k_{m}=k}\left(\begin{array}{c}
k \\
k_{1}, \cdots, k_{m}
\end{array}\right) a_{1}^{k_{1}} \cdots a_{m}^{k_{m}} \cdot I^{k_{1}\left(\alpha_{1}-\alpha_{0}\right)+\cdots+k_{m}\left(\alpha_{m}-\alpha_{0}\right)+\alpha} f(x) \\
+\sum_{k=0}^{\infty}(-1)^{k} \sum_{k_{1}+\cdots+k_{m}=k}\left(\begin{array}{c}
k \\
k_{1}, \cdots, k_{m}
\end{array}\right) \sum_{i=1}^{m} a_{1}^{k_{1}} \cdots a_{i}^{k_{i}+1} \cdots a_{m}^{k_{m}} \\
\cdot I^{k_{1}\left(\alpha_{1}-\alpha_{0}\right)+\cdots+\left(k_{i}+1\right)\left(\alpha_{i}-\alpha_{0}\right)+\cdots+k_{m}\left(\alpha_{m}-\alpha_{0}\right)+\alpha} f(x)=0,
\end{gathered}
$$


after the sign changes and cancellations. Obviously, it is true that

$$
\begin{gathered}
(-1)^{1} \sum_{k_{1}+\cdots+k_{m}=1}\left(\begin{array}{c}
1 \\
k_{1}, \cdots, k_{m}
\end{array}\right) a_{1}^{k_{1}} \cdots a_{m}^{k_{m}} \cdot I^{k_{1}\left(\alpha_{1}-\alpha_{0}\right)+\cdots+k_{m}\left(\alpha_{m}-\alpha_{0}\right)+\alpha} f(x) \\
+(-1)^{0} \sum_{k_{1}+\cdots+k_{m}=0}\left(\begin{array}{c}
0 \\
k_{1}, \cdots, k_{m}
\end{array}\right) \sum_{i=1}^{m} a_{1}^{k_{1}} \cdots a_{i}^{k_{i}+1} \cdots a_{m}^{k_{m}} \\
\cdot I^{k_{1}\left(\alpha_{1}-\alpha_{0}\right)+\cdots+\left(k_{i}+1\right)\left(\alpha_{i}-\alpha_{0}\right)+\cdots+k_{m}\left(\alpha_{m}-\alpha_{0}\right)+\alpha} f(x)=0 .
\end{gathered}
$$

This completes the proof of Theorem 1.

As an example, we can deduce that the following integral equation:

$$
I^{0.5} u(x)+I u(x)+I^{1.5} u(x)=\frac{1}{2} x^{2}
$$

has a unique solution given by

$$
u(x)=\sum_{k=0}^{\infty}(-1)^{k} \sum_{k_{1}+k_{2}=k}\left(\begin{array}{c}
k \\
k_{1}, k_{2}
\end{array}\right) \frac{x^{0.5 k_{1}+k_{2}+1.5}}{\Gamma\left(0.5 k_{1}+k_{2}+2.5\right)},
$$

in the space $L[0, T]$ by using the relation:

$$
\frac{1}{2} x^{2}=\Phi_{3}(x)=\Phi_{2} * \Phi_{1}=I^{2} 1
$$

and Theorem 1.

Using Banach's fixed point theorem, we are now ready to show the uniqueness of solutions to Equation (2) in the space $L[0, T]$.

Theorem 2. Assume that $0 \leqq \alpha_{0}<\alpha_{i}$ for $i=1,2, \cdots, m$ and $\alpha_{0} \leqq \alpha$. Suppose also that there exists a constant $C \geqq 0$ such that

$$
\left|g\left(x, y_{1}\right)-g\left(x, y_{2}\right)\right| \leqq C\left|y_{1}-y_{2}\right|,
$$

for all $x \in[0, T], y_{1}, y_{2} \in \mathbb{R}, g(x, 0) \in L[0, T]$ and

$$
C T^{\alpha-\alpha_{0}} E_{\left(\alpha_{1}-\alpha_{0}, \cdots, \alpha_{m}-\alpha_{0}\right), \alpha-\alpha_{0}+1}\left(\left|a_{1}\right| T^{\alpha_{1}-\alpha_{0}}, \ldots,\left|a_{m}\right| T^{\alpha_{m}-\alpha_{0}}\right)<1 .
$$

Then the Equation (2) has a unique solution in the space $L[0, T]$.

Proof. Let $u \in L[0, T]$. Then $g(x, u(x)) \in L[0, T]$. Indeed, we have

$$
\begin{aligned}
& |g(x, u(x))|=|g(x, u(x))-g(x, 0)+g(x, 0)| \leqq|g(x, u(x))-g(x, 0)|+|g(x, 0)| \\
& \leqq C|u(x)|+|g(x, 0)|,
\end{aligned}
$$

which implies that

$$
\int_{0}^{T}|g(x, u(x))| d x \leqq C \int_{0}^{T}|u(x)| d x+\int_{0}^{T}|g(x, 0)| d x<\infty .
$$

We now define a nonlinear mapping $S$ on $L[0, T]$ as follows:

$$
\begin{aligned}
& (S u)(x)=\sum_{k=0}^{\infty}(-1)^{k} \sum_{k_{1}+\cdots+k_{m}=k}\left(\begin{array}{c}
k \\
k_{1}, \cdots, k_{m}
\end{array}\right) a_{1}^{k_{1}} \cdots a_{m}^{k_{m}} \\
& \cdot I^{k_{1}\left(\alpha_{1}-\alpha_{0}\right)+\cdots+k_{m}\left(\alpha_{m}-\alpha_{0}\right)+\alpha-\alpha_{0}} g(x, u(x)) \text {. }
\end{aligned}
$$


It follows from the lines of the proof of Theorem 1 that

$$
\begin{aligned}
\|S(u)\| \leqq & T^{\alpha-\alpha_{0}} E_{\left(\alpha_{1}-\alpha_{0}, \cdots, \alpha_{m}-\alpha_{0}\right), \alpha-\alpha_{0}+1}\left(\left|a_{1}\right| T^{\alpha_{1}-\alpha_{0}}, \cdots,\left|a_{m}\right| T^{\alpha_{m}-\alpha_{0}}\right) \\
& \cdot\|g(x, u(x))\|<\infty,
\end{aligned}
$$

which shows that $S$ is a mapping from $L[0, T]$ to itself.

It now remains to show that the mapping $S$ is contractive. In fact, we have

$$
\int_{0}^{T}|g(x, u(x))-g(x, v(x))| d x \leqq C \int_{0}^{T}|u(x)-v(x)| d x
$$

and

$$
\begin{aligned}
\| S(u)- & S(v) \| \leqq T^{\alpha-\alpha_{0}} E_{\left(\alpha_{1}-\alpha_{0}, \cdots, \alpha_{m}-\alpha_{0}\right), \alpha-\alpha_{0}+1}\left(\left|a_{1}\right| T^{\alpha_{1}-\alpha_{0}}, \cdots,\left|a_{m}\right| T^{\alpha_{m}-\alpha_{0}}\right) \\
& \cdot\|g(x, u(x))-g(x, v(x))\| \\
& \leqq C T^{\alpha-\alpha_{0}} E_{\left(\alpha_{1}-\alpha_{0}, \cdots, \alpha_{m}-\alpha_{0}\right), \alpha-\alpha_{0}+1}\left(\left|a_{1}\right| T^{\alpha_{1}-\alpha_{0}}, \cdots,\left|a_{m}\right| T^{\alpha_{m}-\alpha_{0}}\right)\|u-v\| \\
& =q\|u-v\|,
\end{aligned}
$$

where

$$
q=C T^{\alpha-\alpha_{0}} E_{\left(\alpha_{1}-\alpha_{0}, \cdots, \alpha_{m}-\alpha_{0}\right), \alpha-\alpha_{0}+1}\left(\left|a_{1}\right| T^{\alpha_{1}-\alpha_{0}}, \ldots,\left|a_{m}\right| T^{\alpha_{m}-\alpha_{0}}\right)<1 .
$$

This completes the proof of Theorem 2.

Finally, we present the sufficient conditions for the uniqueness of the solution of Equation (3) in the product space $L[0, T] \times L[0, T]$.

Theorem 3. Assume that $0 \leqq \alpha_{0}<\alpha_{i}, 0 \leqq \beta_{0}<\beta_{i}$ for $i=1,2, \cdots$, $m$ and $\alpha_{0} \leqq \alpha, \beta_{0} \leqq \beta$. Suppose also that there exist constants $C_{1}, C_{2}, C_{3}$ and $C_{4}$ such that

$$
\left|g_{1}\left(x, y_{1}, y_{2}\right)-g_{1}\left(x, z_{1}, z_{2}\right)\right| \leqq C_{1}\left|y_{1}-z_{1}\right|+C_{2}\left|y_{2}-z_{2}\right|
$$

and

$$
\left|g_{2}\left(x, y_{1}, y_{2}\right)-g_{2}\left(x, z_{1}, z_{2}\right)\right| \leqq C_{3}\left|y_{1}-z_{1}\right|+C_{4}\left|y_{2}-z_{2}\right|,
$$

for all $x \in[0, T], y_{1}, y_{2}, z_{1}, z_{2} \in \mathbb{R}, g_{1}(x, 0,0), g_{2}(x, 0,0) \in L[0, T]$ and

$$
\begin{aligned}
q=\max & \left\{C_{1}, C_{2}\right\} T^{\alpha-\alpha_{0}} E_{\left(\alpha_{1}-\alpha_{0}, \cdots, \alpha_{m}-\alpha_{0}\right), \alpha-\alpha_{0}+1}\left(\left|a_{1}\right| T^{\alpha_{1}-\alpha_{0}}, \cdots,\left|a_{m}\right| T^{\alpha_{m}-\alpha_{0}}\right) \\
& +\max \left\{C_{3}, C_{4}\right\} T^{\beta-\beta_{0}} E_{\left(\beta_{1}-\beta_{0}, \cdots, \beta_{m}-\beta_{0}\right), \beta-\beta_{0}+1} \\
& \quad \cdot\left(\left|b_{1}\right| T^{\beta_{1}-\beta_{0}}, \cdots,\left|b_{m}\right| T^{\beta_{m}-\beta_{0}}\right) \\
< &
\end{aligned}
$$

Then, Equation (3) has a unique solution in the space $L[0, T] \times L[0, T]$.

Proof. Let $u, v \in L[0, T]$. Then $g_{1}(x, u(x), v(x)) \in L[0, T]$. Indeed, we have

$$
\begin{aligned}
& \left|g_{1}(x, u(x), v(x))\right| \\
& \quad=\left|g_{1}(x, u(x), v(x))-g_{1}(x, 0,0)+g_{1}(x, 0,0)\right| \\
& \quad \leqq\left|g_{1}(x, u(x), v(x))-g_{1}(x, 0,0)\right|+\left|g_{1}(x, 0,0)\right| \\
& \quad \leqq C_{1}|u(x)|+C_{2}|v(x)|+\left|g_{1}(x, 0,0)\right| .
\end{aligned}
$$


This implies that

$$
\begin{aligned}
\int_{0}^{T} \mid g_{1} & (x, u(x), v(x)) \mid d x \\
& \leqq C_{1} \int_{0}^{T}|u(x)| d x+C_{2} \int_{0}^{T}|v(x)| d x+\int_{0}^{T}|g(x, 0,0)| d x \\
& <\infty
\end{aligned}
$$

Similarly, we can see that $g_{2}(x, u(x), v(x)) \in L[0, T]$.

Let us now define the mappings $S_{1}, S_{2}$ on $L[0, T] \times L[0, T]$ as follows:

$$
\begin{aligned}
S_{1}(u, v)(x)=\sum_{k=0}^{\infty}(-1)^{k} \sum_{k_{1}+\cdots+k_{m}=k}\left(\begin{array}{c}
k \\
k_{1}, \cdots, k_{m}
\end{array}\right) a_{1}^{k_{1}} \cdots a_{m}^{k_{m}} \\
\cdot I^{k_{1}\left(\alpha_{1}-\alpha_{0}\right)+\cdots+k_{m}\left(\alpha_{m}-\alpha_{0}\right)+\alpha-\alpha_{0}} g_{1}(x, u(x), v(x))
\end{aligned}
$$

and

$$
\begin{aligned}
& S_{2}(u, v)(x)=\sum_{k=0}^{\infty}(-1)^{k} \sum_{k_{1}+\cdots+k_{m}=k}\left(\begin{array}{c}
k \\
k_{1}, \cdots, k_{m}
\end{array}\right) a_{1}^{k_{1}} \cdots a_{m}^{k_{m}} \\
& \cdot I^{k_{1}\left(\alpha_{1}-\alpha_{0}\right)+\cdots+k_{m}\left(\alpha_{m}-\alpha_{0}\right)+\alpha-\alpha_{0}} g_{2}(x, u(x), v(x)) \text {. }
\end{aligned}
$$

Furthermore, we define a mapping $S$ on $L[0, T] \times L[0, T]$ as follows:

$$
S(u, v)=\left(S_{1}(u, v), S_{2}(u, v)\right)
$$

with

$$
\|S(u, v)\|=\left\|S_{1}(u, v)\right\|+\left\|S_{2}(u, v)\right\| .
$$

Thus, clearly, we see that

$$
\begin{aligned}
\left\|S_{1}(u, v)\right\| & \leqq T^{\alpha-\alpha_{0}} E_{\left(\alpha_{1}-\alpha_{0}, \cdots, \alpha_{m}-\alpha_{0}\right), \alpha-\alpha_{0}+1}\left(\left|a_{1}\right| T^{\alpha_{1}-\alpha_{0}}, \cdots,\left|a_{m}\right| T^{\alpha_{m}-\alpha_{0}}\right) \\
& \quad \cdot\left\|g_{1}(x, u(x), v(x))\right\| \\
& <\infty
\end{aligned}
$$

and

$$
\begin{aligned}
\left\|S_{2}(u, v)\right\| & \leqq T^{\beta-\beta_{0}} E_{\left(\beta_{1}-\beta_{0}, \cdots, \beta_{m}-\beta_{0}\right), \beta-\beta_{0}+1}\left(\left|b_{1}\right| T^{\beta_{1}-\beta_{0}}, \cdots,\left|b_{m}\right| T^{\beta_{m}-\beta_{0}}\right) \\
& \quad \cdot\left\|g_{2}(x, u(x), v(x))\right\| \\
& <\infty
\end{aligned}
$$

This implies that $S$ is a mapping from $L[0, T] \times L[0, T]$ to itself. It remains to be shown that $S$ is contractive. Indeed, we have

$$
\begin{aligned}
& \left\|S\left(u_{1}, v_{1}\right)-S\left(u_{2}, v_{2}\right)\right\| \\
& =\left\|S_{1}\left(u_{1}, v_{1}\right)-S_{1}\left(u_{2}, v_{2}\right)\right\| \\
& \quad+\left\|S_{2}\left(u_{1}, v_{1}\right)-S_{2}\left(u_{2}, v_{2}\right)\right\|,
\end{aligned}
$$


and

$$
\begin{aligned}
& \left\|S_{1}\left(u_{1}, v_{1}\right)-S_{1}\left(u_{2}, v_{2}\right)\right\| \\
& \leqq T^{\alpha-\alpha_{0}} E_{\left(\alpha_{1}-\alpha_{0}, \cdots, \alpha_{m}-\alpha_{0}\right), \alpha-\alpha_{0}+1}\left(\left|a_{1}\right| T^{\alpha_{1}-\alpha_{0}}, \ldots,\left|a_{m}\right| T^{\alpha_{m}-\alpha_{0}}\right) \\
& \quad \cdot\left\|g_{1}\left(x, u_{1}, v_{1}\right)-g_{1}\left(x, u_{2}, v_{2}\right)\right\| \\
& \leqq T^{\alpha-\alpha_{0}} E_{\left(\alpha_{1}-\alpha_{0}, \cdots, \alpha_{m}-\alpha_{0}\right), \alpha-\alpha_{0}+1}\left(\left|a_{1}\right| T^{\alpha_{1}-\alpha_{0}}, \cdots,\left|a_{m}\right| T^{\alpha_{m}-\alpha_{0}}\right) \\
& \quad \cdot\left(C_{1} \int_{0}^{T}\left|u_{1}(x)-u_{2}(x)\right| d x+C_{2} \int_{0}^{T}\left|v_{1}(x)-v_{2}(x)\right| d x\right) \\
& \leqq \\
& \max \left\{C_{1}, C_{2}\right\} T^{\alpha-\alpha_{0}} E_{\left(\alpha_{1}-\alpha_{0}, \cdots, \alpha_{m}-\alpha_{0}\right), \alpha-\alpha_{0}+1}\left(\left|a_{1}\right| T^{\alpha_{1}-\alpha_{0}}, \cdots,\left|a_{m}\right| T^{\alpha_{m}-\alpha_{0}}\right) \\
& \quad \cdot\left\|\left(u_{1}, v_{1}\right)-\left(u_{2}, v_{2}\right)\right\| .
\end{aligned}
$$

Similarly, we obtain

$$
\begin{aligned}
& \left\|S_{2}\left(u_{1}, v_{1}\right)-S_{2}\left(u_{2}, v_{2}\right)\right\| \\
& \leqq \\
& \qquad \max \left\{C_{3}, C_{4}\right\} T^{\beta-\beta_{0}} E_{\left(\beta_{1}-\beta_{0}, \cdots, \beta_{m}-\beta_{0}\right), \beta-\beta_{0}+1}\left(\left|b_{1}\right| T^{\beta_{1}-\beta_{0}}, \cdots,\left|b_{m}\right| T^{\beta_{m}-\beta_{0}}\right) \\
& \quad \cdot\left\|\left(u_{1}, v_{1}\right)-\left(u_{2}, v_{2}\right)\right\| .
\end{aligned}
$$

We thus find that

$$
\begin{aligned}
& \left\|S\left(u_{1}, v_{1}\right)-S\left(u_{2}, v_{2}\right)\right\| \\
& \leqq \\
& \quad \max \left\{C_{1}, C_{2}\right\} T^{\alpha-\alpha_{0}} E_{\left(\alpha_{1}-\alpha_{0}, \cdots, \alpha_{m}-\alpha_{0}\right), \alpha-\alpha_{0}+1}\left(\left|a_{1}\right| T^{\alpha_{1}-\alpha_{0}}, \ldots,\left|a_{m}\right| T^{\alpha_{m}-\alpha_{0}}\right) \\
& \quad \cdot\left\|\left(u_{1}, v_{1}\right)-\left(u_{2}, v_{2}\right)\right\| \\
& \quad+\max \left\{C_{3}, C_{4}\right\} T^{\beta-\beta_{0}} E_{\left(\beta_{1}-\beta_{0}, \cdots, \beta_{m}-\beta_{0}\right), \beta-\beta_{0}+1}\left(\left|b_{1}\right| T^{\beta_{1}-\beta_{0}}, \ldots,\left|b_{m}\right| T^{\beta_{m}-\beta_{0}}\right) \\
& \quad \cdot\left\|\left(u_{1}, v_{1}\right)-\left(u_{2}, v_{2}\right)\right\| \\
& =q\left\|\left(u_{1}, v_{1}\right)-\left(u_{2}, v_{2}\right)\right\|,
\end{aligned}
$$

where $q$ is defined above and $q<1$. This completes the proof of Theorem 3 .

\section{An Illustrative Example}

In this section, we present the following example to illustrate the use of Theorem 3.

Example 1. The integral system given by

$$
\left\{\begin{array}{l}
I^{0.5} u(x)-I^{1.6} u(x)+I^{1.5} u(x)=\frac{1}{8} I^{1.7} \sin v(x) \\
I^{2.3} v(x)+I^{3.3} v(x)-I^{3.4} v(x)-I^{3.5} v(x)=\frac{1}{53} I^{2.3} \cos u(x),
\end{array}\right.
$$

has a unique solution in $L[0,1] \times L[0,1]$.

Demonstration of Example 1 Clearly, we have

$$
g_{1}(x, u(x), v(x))=\frac{1}{8} \sin v(x)
$$

and

$$
g_{2}(x, u(x), v(x))=\frac{1}{53} \cos u(x),
$$

and $C_{1}=0, C_{2}=1 / 8, C_{3}=1 / 53$ and $C_{4}=0$, by noting that

$$
\left|\sin z_{1}-\sin z_{2}\right| \leqq\left|z_{1}-z_{2}\right|
$$


and

$$
\left|\cos z_{1}-\cos z_{2}\right| \leqq\left|z_{1}-z_{2}\right|,
$$

for all $z_{1}, z_{2} \in \mathbb{R}$. Furthermore, we have

$$
T=1 \quad \text { and } \quad\left|a_{1}\right|=\left|a_{2}\right|=\left|b_{1}\right|=\left|b_{2}\right|=\left|b_{3}\right|=1 .
$$

Hence, we get

$$
\begin{aligned}
q= & \max \left\{C_{1}, C_{2}\right\} T^{\alpha-\alpha_{0}} E_{\left(\alpha_{1}-\alpha_{0}, \cdots, \alpha_{m}-\alpha_{0}\right), \alpha-\alpha_{0}+1}\left(\left|a_{1}\right| T^{\alpha_{1}-\alpha_{0}}, \cdots,\left|a_{m}\right| T^{\alpha_{m}-\alpha_{0}}\right) \\
& \quad+\max \left\{C_{3}, C_{4}\right\} T^{\beta-\beta_{0}} E_{\left(\beta_{1}-\beta_{0}, \cdots, \beta_{m}-\beta_{0}\right), \beta-\beta_{0}+1}\left(\left|b_{1}\right| T^{\beta_{1}-\beta_{0}}, \cdots,\left|b_{m}\right| T^{\beta_{m}-\beta_{0}}\right) \\
= & \frac{1}{8} E_{(1.1,1), 2.2}(1,1)+\frac{1}{53} E_{(1,1.1,1.2), 1}(1,1,1) .
\end{aligned}
$$

It is now evident that

$$
\begin{aligned}
E_{(1.1,1), 2.2}(1,1) \\
=\sum_{k=0}^{\infty} \sum_{k_{1}+k_{2}=k}\left(\begin{array}{c}
k \\
k_{1}, k_{2}
\end{array}\right) \frac{1}{\Gamma\left(1.1 k_{1}+k_{2}+2.2\right)} \\
\quad \leqq \sum_{k=0}^{\infty} \sum_{k_{1}+k_{2}=k}\left(\begin{array}{c}
k \\
k_{1}, k_{2}
\end{array}\right) \frac{1}{\Gamma(k+2)}=\sum_{k=0}^{\infty} \frac{2^{k}}{(k+1) !} \\
=1+\frac{2}{2 !}+\frac{2 \cdot 2}{1 \cdot 2 \cdot 3}+\frac{2 \cdot 2 \cdot 2}{1 \cdot 2 \cdot 3 \cdot 4}+\frac{2 \cdot 2 \cdot 2 \cdot 2}{1 \cdot 2 \cdot 3 \cdot 4 \cdot 5}+\frac{2 \cdot 2 \cdot 2 \cdot 2 \cdot 2}{1 \cdot 2 \cdot 3 \cdot 4 \cdot 5 \cdot 6}+\cdots \\
\leqq \\
\quad 1+1+1+\frac{2}{15}\left(1+\frac{2}{5}+\left(\frac{2}{5}\right)^{2}+\cdots\right) \\
=3+\frac{2}{15} \frac{1}{1-\frac{2}{5}} \leqq 3.23 .
\end{aligned}
$$

On the other hand, we have

$$
E_{(1,1.1,1.2), 1}(1,1,1)=\sum_{k=0}^{\infty} \sum_{k_{1}+k_{2}+k_{3}=k}\left(\begin{array}{c}
k \\
k_{1}, k_{2}, k_{3}
\end{array}\right) \frac{1}{\Gamma\left(k_{1}+1.1 k_{2}+1.2 k_{3}+1\right)} .
$$

It follows from [21] that

$$
\frac{1}{\Gamma\left(k_{1}+1.1 k_{2}+1.2 k_{3}+1\right)} \leqq \frac{1}{\Gamma(k+1)}
$$

for all $k=0,1, \cdots$. This implies that

$$
\begin{aligned}
E_{(1,1.1,1.2), 1}(1,1,1) \\
\quad \leqq \sum_{k=0}^{\infty} \frac{3^{k}}{k !} \\
\quad=1+\frac{3}{1}+\frac{3 \cdot 3}{1 \cdot 2}+\frac{3 \cdot 3 \cdot 3}{1 \cdot 2 \cdot 3}+\frac{3 \cdot 3 \cdot 3 \cdot 3}{1 \cdot 2 \cdot 3 \cdot 4}+\frac{3 \cdot 3 \cdot 3 \cdot 3 \cdot 3}{1 \cdot 2 \cdot 3 \cdot 4 \cdot 5}+\cdots \\
\quad \leqq 1+3+4.5+4.5+3.375+2.025+\left(\frac{1}{80}+1\right)+\frac{1}{2}+\frac{1}{2^{2}}+\frac{1}{2^{3}}+\cdots \\
\quad=20.4125 .
\end{aligned}
$$

Clearly, therefore, we get $q<1$. This completes our demonstration of Example 1 . 


\section{Conclusions}

By using Babenko's approach, Banach's contraction principle and the multivariate Mittag-Leffler function, we have studied several generalized forms of Abel's integral equations and a related coupled system with constant coefficients in Banach spaces. The results, which we have presented in this article, are new and provide interesting generalizations of the existing results in the literature. We have also included some examples, including one example that shows the application of our main theorem (Theorem 3).

Author Contributions: Conceptualization, C.L. and H.M.S.; methodology, C.L.; software, C.L. and H.M.S.; validation, C.L. and H.M.S.; formal analysis, C.L.; investigation, C.L. and H.M.S.; resources, C.L. and H.M.S.; writing —original draft preparation, C.L.; writing—review and editing, C.L. and H.M.S.; visualization, C.L. and H.M.S. All authors have read and agreed to the published version of the manuscript.

Funding: This work is supported by the Natural Sciences and Engineering Research Council of Canada (Grant No. 2019-03907).

Institutional Review Board Statement: Not applicable.

Informed Consent Statement: Not applicable.

Data Availability Statement: Not applicable.

Acknowledgments: The authors are grateful to the two reviewers for their careful reading of the paper with productive comments and suggestions.

Conflicts of Interest: The authors declare no conflict of interest.

\section{References}

1. Samko, S.G.; Kilbas, A.A.; Marichev, O.I. Fractional Integrals and Derivatives: Theory and Applications; Gordon and Breach: Tokyo, Japan; Paris, France; Berlin, Germany; Langhorne, PA, USA, 1993.

2. Kilbas, A.A.; Srivastava, H.M.; Trujillo, J.J. Theory and Applications of Fractional Differential Equations; North-Holland Mathematical Studies; Elsevier (North-Holland) Science Publishers: Amsterdam, The Netherlands; London, UK; New York, NY, USA, 2006; Volume 204.

3. Hille, E.; Tamarkin, J.D. On the theory of linear integral equations. Ann. Math. 1930, 31, 479-528. [CrossRef]

4. Gorenflo, R.S.; Vessela, S. Abel Integral Equations; Analysis and Applications; Lecture Notes in Mathematics; Springer: Berlin/Heidelberg, Germany; New York, NY, USA; London, UK, 1991; Volume 1461.

5. Srivastava, H.M.; Buschman, R.G. Theory and Applications of Convolution Integral Equations; Kluwer Series on Mathematics and Its Applications; Kluwer Academic Publishers: Dordrecht, The Netherlands; Boston, MA, USA; London, UK, $1992 ;$ Volume 79.

6. Raina, R.K.; Srivastava, H.M.; Kilbas, A.A.; Saigo, M. Solvability of some Abel-type integral equations involving the Gauss hypergeometric function as kernels in the spaces of summable functions. ANZIAM J. 2001, 43, 291-320. [CrossRef]

7. Podlubny, I. Fractional Differential Equations: An Introduction to Fractional Derivatives, Fractional Differential Equations, to Methods of Their Solution and Some of Their Applications, Mathematics in Science and Engineering; Academic Press: New York, NY, USA; London, UK; Sydney, Australia; Tokyo, Japan; Toronto, ON, Canada, 1999; Volume 198.

8. Li, C.; Beaudin, J. Uniqueness of Abel's integral equations of the second kind with variable coefficients. Symmetry 2021, $13,1064$. [CrossRef]

9. Li, C.; Plowman, H. Solutions of the generalized Abel's integral equations of the second kind with variable coefficients. Axioms 2019, 8, 137. [CrossRef]

10. Pskhu, A. On solution representation of generalized Abel integral equation. J. Math. 2013, 2013, 106251. [CrossRef]

11. Gorenflo, R.; Luchko, Y. Operational method for solving generalized Abel integral equation of second kind. Integral Transform. Spec. Funct. 1997, 5, 47-58. [CrossRef]

12. Haubold, H.J.; Mathai, A.M.; Saxena, R.K. Mittag-Leffler functions and their applications. J. Appl. Math. 2011, $2011,298628$. [CrossRef]

13. Li, C.; Clarkson, K. Babenko's approach to Abel's integral equations. Mathematics 2018, 6, 32. [CrossRef]

14. Pskhu, A. Solution of a multidimensional Abel integral equation of the second kind with partial integrals. Differ. Equ. 2017, 53, 1160-1164. [CrossRef]

15. Mohamed, M.S.; Gepreel, K.A.; Al-Malki, F.A.; Al-Humyani, M. Approximate solutions of the generalized Abel's integral equations using the extension Khan's homotopy analysis transformation method. J. Appl. Math. 2015, 2015, 357861. [CrossRef]

16. Brunner, H.; Crisci, M.R.; Russo, E.; Vecchio, A. A family of methods for Abel integral equations of the second kind. J. Comput. Appl. Math. 1991, 34, 211-219. [CrossRef] 
17. Hadid, S.B.; Luchko, Y. An operational method for solving fractional differential equations of an arbitrary real order. Panamer. Math. J. 1996, 6, 57-73.

18. Babenkos, Y.I. Heat and Mass Transfer; Khimiya: Leningrad, Russia, 1986. (In Russian)

19. Li, C. Several results of fractional derivatives in $\mathcal{D}^{\prime}\left(R_{+}\right)$. Fract. Calc. Appl. Anal. 2015, 18, 192-207. [CrossRef]

20. Barros-Neto, J. An Introduction to the Theory of Distributions; Marcel Dekker Incorporated: New York, NY, USA, 1973.

21. Gradshteyn, I.S.; Ryzhik, I.M. Tables of Integrals, Series, and Products; Academic Press: New York, NY, USA, 1980. 